\title{
CHARACTERIZATION OF UNSTEADY FLOW STRUCTURES AROUND TANDEM CYLINDERS FOR COMPONENT INTERACTION STUDIES IN AIRFRAME NOISE
}

\author{
Luther N. Jenkins* , Mehdi R. Khorrami ${ }^{\dagger}$, Meelan M. Choudhari ${ }^{\dagger}$, and Catherine B. McGinley* \\ NASA Langley Research Center, Hampton, Virginia, 23681
}

A joint computational and experimental study has been performed at NASA Langley Research Center to investigate the unsteady flow generated by the components of an aircraft landing gear system. Because the flow field surrounding a full landing gear is so complex, the study was conducted on a simplified geometry consisting of two cylinders in tandem arrangement to isolate and characterize the pertinent flow phenomena. This paper focuses on the experimental effort where surface pressures, 2-D Particle Image Velocimetry, and hot-wire anemometry were used to document the flow interaction around the two cylinders at a Reynolds Number of $1.66 \times 10^{5}$, based on cylinder diameter, and cylinder spacing-todiameter ratios, L/D, of 1.435 and 3.70. Transition strips were applied to the forward cylinder to produce a turbulent boundary layer upstream of the flow separation. For these flow conditions and L/D ratios, surface pressures on both the forward and rear cylinders show the effects of L/D on flow symmetry, base pressure, and the location of flow separation and attachment. Mean velocities and instantaneous vorticity obtained from the PIV data are used to examine the flow structure between and aft of the cylinders. Shedding frequencies and spectra obtained using hot-wire anemometry are presented. These results are compared with unsteady, Reynolds-Averaged Navier-Stokes (URANS) computations for the same configuration in a companion paper by Khorrami, Choudhari, Jenkins, and McGinley (2005). The experimental dataset produced in this study provides information to better understand the mechanisms associated with component interaction noise, develop and validate time-accurate computer methods used to calculate the unsteady flow field, and assist in modeling of the radiated noise from landing gears.

\section{Nomenclature}

$D \quad=$ cylinder diameter, 0.04445 meters

$C_{P} \quad=$ pressure coefficient, $\frac{p-p_{\infty}}{q_{\infty}}$

$L \quad=$ distance between cylinder centers, meters

$p \quad=$ pressure measured along model surface, $\mathrm{Pa}$

$p_{\infty} \quad=$ freestream static pressure, $\mathrm{Pa}$

$q_{\infty} \quad=$ freestream dynamic pressure, $\mathrm{Pa}$

$S t \quad=$ Strouhal Number, $\frac{f D}{U_{\infty}}$

TKE $=$ turbulent kinetic energy

$U=$ streamwise velocity component, $\mathrm{m} / \mathrm{s}$

$U_{\infty} \quad=$ freestream velocity, $\mathrm{m} / \mathrm{s}$

$V \quad=$ vertical velocity component, $\mathrm{m} / \mathrm{s}$

$X=$ streamwise coordinate, $\mathrm{mm}$

\footnotetext{
* Research Engineer, Flow Physics and Control Branch

${ }^{\dagger}$ Research Engineer, Computational Aerosciences Branch, Associate Fellow, AIAA
} 


\section{Introduction}

In its quest to reduce airframe noise, NASA's Quiet Aircraft Technology (QAT) project has conducted systematic studies on the flap side edge, slat, and landing gear to better understand the noise generation process and develop noise reduction techniques. Significant progress has been made in understanding the noise source mechanisms associated with the flap and the slat. However, the landing gear, which has been identified as a major contributor to airframe noise on large aircraft during approach, presents a significant challenge for noise prediction methods. The unsteady flow around its numerous components, some of which are depicted in Figure 1, and the interaction of unsteady flow among other components of the landing gear generate noise over a broad range of frequencies. To identify and assess the effect of flow interactions on the overall noise levels, additional insight and details about such flow fields are needed.

Several current approaches for noise prediction on landing gears rely on an understanding of the unsteady flow and its interaction with other components. The Aircraft Noise Prediction Program (ANOPP) ${ }^{1}$ models the dimensions of the wheels and the length of the oleo strut to predict the size of the turbulent wake. This information, along with a database consisting of flight and wind tunnel data, is used as inputs into an empirical formulation for landing gear noise. Another approach leverages techniques developed for the flap side and slat: namely, using a time-accurate flow solver to compute the unsteady flow field around the landing gear and then using the unsteady flow field to compute the far-field noise ${ }^{2}$. The newest method is called the Landing Gear Model and Acoustic Prediction (LGMAP) ${ }^{3}$ which represents the landing gear as a number of acoustic elements. Each element is modeled as a cylinder and the radiated noise is computed using geometric parameters, the upstream and downstream environments, and a loading spectrum with a peak frequency corresponding to the shedding Strouhal number for the cylinder. Noise radiated from each of the elements can then be aggregated to determine the total noise. The latter two approaches lend themselves to building block configurations that can be used to assess and validate modeling techniques for flow interactions.

A canonical problem to advance modeling techniques for flow interactions corresponds to the tandem cylinder arrangement. Tandem cylinders with similar diameters can be found in several locations on a landing gear, such as multiple wheels, axles, and hydraulic lines. Additionally, there exist similar arrangements of cylinders with different diameters, such as the combination of the main strut and adjacent hydraulic lines. Numerous experimental and computational studies have been conducted on the tandem configuration due to its practical application in systems used for cooling, venting, and platform support. In most cases, the studies were performed at Reynolds Numbers less than $1.5 \times 10^{5}$ (subcritical) and focused on fundamental issues such as boundary layer development, numerical prediction of flow interference, flow-induced vibrations, and characterization of the wake structure for various cylinder spacing ${ }^{4-7}$. According to Reference 8 , several flow patterns have been observed based on the separation distance between the cylinders (L/D). For L/D < 1.1, the two cylinders behave as a single bluff body with vortex shedding occurring at the rear cylinder only. For $1.1<\mathrm{L} / \mathrm{D}<1.6$, the shear layers from the front cylinder attach alternately to the rear cylinder and vortex shedding occurs on the rear cylinder. For $1.6<\mathrm{L} / \mathrm{D}<2.5$, the shear layers from the front cylinder attach to the rear cylinder and vortex shedding occurs on the rear cylinder. For separation distances, $2.5<\mathrm{L} / \mathrm{D}<3.2$, intermittent shedding can be detected in the region between the cylinders and vortex shedding occurs on the rear cylinder. For $3.2<\mathrm{L} / \mathrm{D}<3.8$, the flow between the cylinders is bistable and switches between intermittent shedding and constant shedding. For L/D > 3.8, vortex shedding occurs at both cylinders with the same characteristics as a single cylinder. Similar flow regimes have been identified at higher Reynolds numbers ${ }^{9}$ but, in most cases, only qualitative details about the flow structure are available. Excellent quantitative details about the time-averaged and instantaneous flow structure for several of the flow regimes described above have been obtained by Lin, et. al. ${ }^{10}$ at $\mathrm{Re}=1.0 \times 10^{4}$ using Particle Image Velocimetry (PIV). Because the Reynolds numbers associated with the landing gear flow field are typically higher than $1.5 \times 10^{5}$, similar data is needed at higher Reynolds numbers for model assessment and validation.

The purpose of the present investigation was to document the unsteady flow field around a tandem cylinder arrangement at a post-critical Reynolds number using PIV and hot-wire anemometry, to determine how the wake of 
the front cylinder interacts with the rear cylinder, and how the wake of the entire configuration is modified by this interaction (compared to a single cylinder). The experimental dataset provides information to better understand the flow features associated with component interaction noise, develop and validate computational techniques for predicting the unsteady flow field, and hence, assist in modeling the radiated noise from landing gears.

\section{Experimental Apparatus and Techniques}

\section{A. Test Facility}

The experiment was conducted in the NASA-Langley Basic Aerodynamics Research Tunnel (BART). This tunnel is a subsonic, atmospheric wind tunnel used to investigate the fundamental characteristics of complex flow fields and to acquire detailed data for the development and validation of computational fluid dynamics (CFD) models and methods. The tunnel has a closed test section with a height of 0.711 meters, a width of 1.016 meters, and a length of 3.048 meters. The test was conducted at a free stream velocity of $56 \mathrm{~m} / \mathrm{s}$, which corresponds to a Reynolds number based on cylinder diameter of $1.66 \times 10^{5}$. The free stream turbulence level at these conditions is $0.09 \%$. Additional information about the BART can be found in References 11-13.

\section{B. Model}

The geometry used in this investigation consisted of two cylinders in tandem arrangement. Figure 2 shows the cylinders installed in the BART and Figure 3 provides a schematic of the model with the coordinate reference system. Each cylinder measured $44.45 \mathrm{~mm}$ in diameter and $709.6 \mathrm{~mm}$ in length and was attached to a base that was inserted into a track mechanism secured to the tunnel floor. With this arrangement, the front cylinder was fixed and the rear cylinder could be translated upstream and downstream to achieve the desired spacing. To accommodate the translation of the rear cylinder and satisfy the optical access requirements for the flow field measurements, spring-loaded, Teflon ${ }^{\circledR}$ caps were inserted into the top of the cylinders to produce a tension fit against the glass ceiling. A guide wire was attached to the top of the upstream cylinder to minimize its deflection under loading. Although measurements were made at several spacings, this paper will focus on two cases: $\mathrm{L} / \mathrm{D}=1.435$ and $\mathrm{L} / \mathrm{D}=$ 3.70. Data on a single cylinder was also acquired for comparisons with the tandem configurations. This was accomplished with the front cylinder in its fixed position and the rear cylinder removed.

Each cylinder was instrumented with lower, center and upper pressure rings located at $228.6 \mathrm{~mm}, 355.6 \mathrm{~mm}$, and $457.2 \mathrm{~mm}$, respectively, from the tunnel floor. The upper and lower rings consist of 18 orifices spaced every 20 degrees around the cylinder circumference, whereas the center ring consists of 36 orifices spaced every 10 degrees around the cylinder circumference. In addition, 9 pressure orifices with $50.8 \mathrm{~mm}$ spacing were installed along the length of each cylinder at $\theta=180^{\circ}$ to monitor pressure recovery and check for axial variations.

To simulate the high Reynolds number flows associated with actual landing gear components, transition strips were attached to the upstream cylinder to produce a turbulent boundary layer. Instead of using wire or grit, the transition strip consisted of "pinked" tape with a nominal thickness of $0.229 \mathrm{~mm}$ applied along the entire length of the cylinder in the region between $50^{\circ}<\theta<60^{\circ}$ and $300^{\circ}<\theta<310^{\circ}$. The tape was positioned with the "straight" edge facing upstream and the "pinked" edge facing downstream. According to References 14 and 15, this type of transition strip is typically attached to the model with the "pinked" edge facing upstream. However, this arrangement did not result in a turbulent separation. To determine the location of the transition strip and assess its effectiveness, the pressure distribution was measured in the present case for a cylinder spacing of L/D = 8.0 and compared to available pressure data at higher Reynolds numbers ${ }^{16}$. As shown in Figure 4, the transition strip produced a pressure distribution that is comparable to that of higher Reynolds numbers, despite slight differences in peak suction and pressure recovery. In addition, the shedding frequency of the upstream cylinder alone (single cylinder) with the transition strip applied was measured using a hot wire and found to be approximately $365 \mathrm{~Hz}$. The Strouhal Number computed using this frequency, the cylinder diameter, and the free stream velocity is 0.290. Strouhal numbers associated with higher Reynolds number flows are typically in this range ${ }^{15}$.

\section{Data Acquisition}

Velocity measurements were made using a 2D, Digital Particle Image Velocimetry (DPIV) system. The system features two, high-resolution, video cameras with a sensor size of 1360 pixels by 1024 pixels. The cameras were mounted to a traverse system surrounding the tunnel along with the laser and light-sheet optics. A $1.5 \mathrm{~mm}$ thick 
light sheet was generated using a pulsed, frequency-doubled, $200 \mathrm{~mJ} \mathrm{Nd-YAG} \mathrm{laser} \mathrm{operated} \mathrm{at} 5 \mathrm{~Hz}$. The light sheet was aligned to be perpendicular to the cylinder surfaces at a location $6 \mathrm{~mm}$ above the center pressure ring. Images were acquired using $50 \mathrm{~mm}$ lenses to obtain a field-of-view for a single camera of approximately $80 \mathrm{~mm}$ wide by $60 \mathrm{~mm}$ high. Two cameras were used so the fields of view were overlapped by to achieve an effective field of view of approximately $156 \mathrm{~mm}$ wide x $60 \mathrm{~mm}$ high. The entire room housing the tunnel was seeded with 1 micron particles produced by a theatrical fog machine. A minimum of 1250 image pairs were acquired for each configuration and processed using the algorithm described in Reference 17 with a 24 pixel by 24 pixel interrogation window and 50\% over sampling to obtain instantaneous velocities. The instantaneous velocities were then used to compute the instantaneous spanwise vorticity and flow statistics. Statistical convergence was confirmed by examining the asymptotic behavior of the first and second moments of the velocity at select points in the flow field where the velocity gradient and turbulence intensities achieved their highest values. The uncertainty in the PIV data is estimated to be $\pm 1.18 \mathrm{~m} / \mathrm{s}$.

Hot-wire anemometry was used to measure the shedding frequencies and determine correlation lengths for the single cylinder and the tandem configurations. To capture the shedding frequencies, a single hot-wire probe was positioned 2.74 diameters downstream of the single or rear cylinder and traversed from Y/D $=0$ to $\mathrm{Y} / \mathrm{D}=-3$. Correlation lengths were determined for the three configurations in a similar fashion using two, hot-wire probes. One probe was fixed at a position of 2.74 diameters downstream of the single or rear cylinder and $\mathrm{Y} / \mathrm{D}=0.685$. The second probe was positioned directly above it and traversed in the spanwise direction until the signals from the two wires were no longer correlated. The hot-wire signals were low-pass filtered at $5 \mathrm{kHz}$ and then acquired using a $12.8 \mathrm{kHz}$ sample rate.

\section{Discussion of Results}

\section{A. Surface Flow Characteristics}

Measurements of the mean surface pressure were used to validate the experimental setup through comparisons with existing data and to examine the effect of cylinder spacing on the peak suction, flow separation and pressure recovery. Figure 5 compares the pressure coefficient measured on the front cylinder for $\mathrm{L} / \mathrm{D}=1.435, \mathrm{~L} / \mathrm{D}=3.70$, and $\mathrm{L} / \mathrm{D}=7.0$ with data acquired for a single cylinder. The cylinder spacing has minimal effect on the pressures along the front cylinder for $\mathrm{L} / \mathrm{D}=7.0$; however, some differences are noted for $\mathrm{L} / \mathrm{D}=1.435$ and $\mathrm{L} / \mathrm{D}=3.70$ at the location of peak suction and in the pressure recovery region. Interestingly, the pressure distributions become asymmetric at the smaller cylinder spacing and show less suction but better pressure recovery due to proximity of the rear cylinder.

Figure 6 shows the pressure distributions measured on the rear cylinder. For $\mathrm{L} / \mathrm{D}=3.70$ and $\mathrm{L} / \mathrm{D}=7.00$, the mean flow attaches at the forward stagnation point and peak suction occurs around $\theta=80^{\circ}$. The disparity in pressure levels near the forward stagnation point is attributed to shedding from the front cylinder and its the interaction with the rear cylinder. For $\mathrm{L} / \mathrm{D}=1.435$, the mean flow from the upstream cylinder attaches to the upper surface of the rear cylinder at $\theta=60^{\circ}$, accelerates, and then detaches around $\theta=120^{\circ}$. Similar features are observed along the lower surface, though the pressure levels are higher and separation occurs further aft on the cylinder. Pressures along the upstream face of the rear cylinder show a slight pressure rise in the region $0^{\circ} \leq \theta \leq 40^{\circ}$ and a pressure decrease in the region $320^{\circ} \leq \theta \leq 360^{\circ}$. These trends are consistent with the asymmetry in the pressure recovery region seen in Figure 5 and suggest an asymmetric gap flow. $\mathrm{Gu}^{9}$ measured a similar pressure distribution for $\mathrm{L} / \mathrm{D}=1.5$ at a post-critical Reynolds number $\left(4.5 \times 10^{5}\right)$ but no hypothesis was provided as to its cause.

\section{B. Off-body Flow Field}

For the purpose of this discussion, the flow field has been divided into two regions: flow between the cylinders and flow downstream of the rear cylinder. The former will be referred to as the gap flow and the latter as the configuration near wake. Although it would be extremely informative to measure both regions simultaneously, this was only feasible for the $\mathrm{L} / \mathrm{D}=1.435$ case. For the flow field data presented, distances have been normalized by the cylinder diameter and are referenced to the center of the front cylinder for the gap flow figures and to the center of the rear cylinder in the configuration near wake figures. All velocities, turbulence quantities, and vorticity have been normalized by the freestream velocity and/or cylinder diameter as appropriate. 


\section{Gap Flow: $L / D=1.435$}

The gap flow is of primary importance because its interaction with the rear cylinder influences the shedding characteristics of the rear cylinder. Figures 7a-e show streamlines, color contours of the streamwise and vertical velocity, turbulent kinetic energy, and spanwise vorticity associated with the mean flow. Figures 7f-h show selected frames of instantaneous spanwise vorticity. Collectively, these images provide significant information about the gap flow. First, the surface streamlines clearly show that the mean flow is not symmetric about $Y / D=0$, despite the fact that the pressure distribution in Figure 6 indicates the mean upper and lower shear layers impinge on the rear cylinder at the same relative locations $\left(\theta=60^{\circ}\right.$ and $\theta=300^{\circ}$, respectively). Although some motion was observed at the shear layer attachment point during an inspection of over 100 PIV frames of instantaneous spanwise vorticity, alternating reattachment on the rear cylinder as described by Zdravkovich ${ }^{8}$ for these cylinder spacings was not evident. After the lower shear layer attaches to the rear cylinder and bifurcates, a portion of the flow travels vertically along the forward surface of the rear cylinder, turns upstream, attaches to the rearward surface of the front cylinder, and then travels down toward the lower shear layer to form a recirculation zone. TKE levels shown in Figure $7 \mathrm{~d}$ are relatively low throughout the gap except for a small region near the upstream surface of the rear cylinder. Based on the mean vorticity contours in Figure 7e, the recirculation does not appear to be associated with a vortex. This flow pattern is consistent with the asymmetry in the pressure distribution on the front cylinder and agrees with the average flow structure described by Lin et. al. ${ }^{10}$ from PIV measurements for a cylinder spacing of $\mathrm{L} / \mathrm{D}=1.5$. Figures $7 \mathrm{f}-\mathrm{h}$ also provide evidence that vortical structures from the shear layer are sometimes convected into the gap and interact with the surfaces of both cylinders.

\section{Gap Flow: $L / D=3.70$}

In contrast to the $\mathrm{L} / \mathrm{D}=1.435$ case, the gap flow for the $\mathrm{L} / \mathrm{D}=3.70$ case shown in Figures $8 \mathrm{a}-\mathrm{h}$ looks very similar to the flow behind a single cylinder except in the immediate vicinity of the rear cylinder. Streamlines in Figure 8a show a recirculating flow region behind the front cylinder whose length, approximately 1.1D, is comparable to the length associated with the single cylinder case discussed in the next section. Other similar features are shown in Figures $8 \mathrm{~b}$ and $8 \mathrm{c}$. These include significant flow towards the centerline from the upper and lower parts of the flow on the order of $20 \%$ of the freestream velocity and high TKE levels (20\%) in the middle of the gap region $(1 \leq \mathrm{X} / \mathrm{D} \leq 2.8)$ with some moderate levels near the upstream face of the rear cylinder. The high levels in the middle of the gap region occur where the recirculation region closes. Moderate TKE levels near the rear cylinder are reflect velocity fluctuations associated with the vortical structures from the shear layer as they pass by the upstream surface of the rear cylinder.

\section{Configuration Near Wake}

For an unsteady flow field, mean data does not provide structural details but can be used to verify the symmetry of the flow field and characterize the flow associated with each case. Given the potential sensitivity of the tandem cylinder flow to configuration asymmetry, especially when the cylinder spacing is small, documenting the meanflow symmetry about the geometric symmetry plane is particularly important. Figures 9a-c show transverse profiles of mean velocity and streamwise turbulence intensity extracted from the PIV data downstream of the single/rear cylinder at $\mathrm{X} / \mathrm{D}=0.75$ and Figures 10a-c show streamwise profiles of mean velocity and TKE along the centerline behind the single/rear cylinder. For the most part, Figures $9 \mathrm{a}-\mathrm{c}$ indicate flow symmetry about $\mathrm{Y} / \mathrm{D}=0$ at this location for the single cylinder and $\mathrm{L} / \mathrm{D}=3.70$; however, the profiles for $\mathrm{L} / \mathrm{D}=1.435$ show considerable asymmetry. The minimum streamwise velocity does not occur at the centerline but rather above it. The magnitude of the vertical velocity is greater below the centerline and the distance from the centerline to the peaks in the turbulent intensity profiles differ by $\mathrm{Y} / \mathrm{D}=0.08$. The streamwise profiles in Figure 10b also show asymmetry for $\mathrm{L} / \mathrm{D}=1.435$. On the centerline, the vertical velocity is expected to be near zero; however, the vertical velocity varies between $6 \%$ and $8 \%$ of the freestream velocity indicating a net flow across the centerline. Although great care was taken to align the tandem configuration with the freestream, it was hypothesized that the asymmetry may be due to misalignment between the two cylinders. To investigate this in more detail, additional calculations were performed after the experiment using CFD for $\mathrm{L} / \mathrm{D}=1.435$ and 3.70 with the configuration rotated 0.5 degrees and 1

degrees with respect to the oncoming flow. The reader is referred to the companion paper by Khorrami, et.al. ${ }^{17}$ for specific details and a more in-depth discussion. In short, the results show that flow angle has a greater impact on the flow field for $\mathrm{L} / \mathrm{D}=1.435$ than for $\mathrm{L} / \mathrm{D}=3.70$. Despite differences in the peak suction levels between $\mathrm{CFD}$ and the experiment, the computed pressure distribution for $\mathrm{L} / \mathrm{D}=1.435$ show the same asymmetry on the front and rear cylinders. Based on these results, it appears that flow angularity, flow non-uniformity, and other tunnel conditions could have a significant impact on the symmetry of the flow field when the cylinder spacing is small. 
In addition to asymmetry, the profiles show other differences between the tandem configurations that are relevant to sound generation. First, Figure 10a shows that the recirculation region for $\mathrm{L} / \mathrm{D}=3.70$ is about 2.5 times smaller than that for $\mathrm{L} / \mathrm{D}=1.435$. A smaller recirculation region behind the rear cylinder may make it more susceptible to pressure fluctuations associated with vortex formation. In fact, the highest turbulent kinetic energy levels shown in Figure 10c for the $\mathrm{L} / \mathrm{D}=3.70$ case occur within $1 \mathrm{D}$ of the rear cylinder and are 5.5 times higher than those for the $\mathrm{L} / \mathrm{D}=1.435$ case in the same region. For $\mathrm{L} / \mathrm{D}=1.435$, the turbulent kinetic energy profile shows no dramatic rise and fall. Instead, it gradually increases to its maximum value of $4 \%$ after 1.5 diameters.

Using the results for the single cylinder as a reference, the contours in Figures 11a-c, 12a-c, 13a-c, and 14a-c highlight the impact of the gap flow on the wake structure. For $\mathrm{L} / \mathrm{D}=1.435$, Figures $11 \mathrm{~b}$ and $12 \mathrm{~b}$ show that the gap flow creates a slightly narrower wake and a region over 3D long where the vortex shedding pulls fluid towards the centerline. Figures $13 \mathrm{~b}$ and $14 \mathrm{~b}$ show that the asymmetry seen in the gap is also evident in the recirculation region and the maximum TKE level is reduced by over $30 \%$. On the other hand, the $\mathrm{L} / \mathrm{D}=3.70$ case shows the opposite trend. In Figures 11c and 12c, the wake broadens and the highest mean vertical velocities are concentrated in a region near the rear cylinder. The streamlines in Figure 13c reveal a much smaller recirculation region and Figure $14 \mathrm{c}$ shows high levels of TKE within $1.5 \mathrm{D}$ of the rear cylinder. The proximity of high levels of TKE to the rear cylinder may increase the impact of the velocity fluctuations, thereby increasing the amplitude of pressure fluctuations on the cylinder surface.

\section{Instantaneous spanwise vorticity}

The instantaneous spanwise vorticity field reveals more details about the structure of the unsteady flow field. Whereas in Figures 15a-c the mean vorticity in the shear layer quickly diffuses within a distance of approximately $1.5 \mathrm{D}$, select color contours of instantaneous spanwise vorticity in Figures 16a-c show that vortical structures generated within the shear layer persist well downstream and help to highlight the wake structure. Vortical structures in the free shear layer have been visualized at much lower Reynolds numbers by Couregelongue ${ }^{19}$ on a single cylinder and measured on a tandem configuration by Lin, et. al. ${ }^{10}$. Figure 17 shows a schematic of what Couregelongue considered to be "transition eddies" associated with an intermediate subcritical flow regime. The pattern is remarkably similar to the results shown in Figures 16a-c although in the case of this experiment, the free shear layer is assumed to be fully turbulent.

The experimental design was not set up to take data at various phases of the shedding process; however, an attempt was made to compute the phase-averaged vorticity by averaging a select group of PIV frames. The criterion for frame selection was the appearance of a vortex resembling a traditional shed vortex in a $12 \mathrm{~mm}$ radius about the point $\mathrm{X} / \mathrm{D}=1.34, \mathrm{Y} / \mathrm{D}=-0.08$. Figure 18 was produced using $20 \mathrm{PIV}$ frames where this condition was satisfied. Compared to Figure 15a, the vorticity does not diffuse as rapidly and a more coherent region of vorticity begins to appear.

\section{Shedding Frequencies and Correlation Lengths}

Hot-wire measurements were made downstream of the single/rear cylinder at $\mathrm{X} / \mathrm{D}=2.74$ and $\mathrm{Y} / \mathrm{D}=-0.685$ to obtain velocity spectra and spanwise correlation lengths. Figure 19 shows the measurement location relative to the cylinder and Figure 20 shows the spectra associated with each configuration. Vortex shedding frequencies and computed Strouhal numbers are listed in Table 1. The spectra for the single cylinder and $\mathrm{L} / \mathrm{D}=1.435$ are comparable in their overall shape and level except at the vortex shedding frequency where the tone for $\mathrm{L} / \mathrm{D}=1.435$ is considerably weaker. The spectrum for $\mathrm{L} / \mathrm{D}=3.70$ shows higher levels overall but a lower vortex shedding frequency. Figure 21 shows that the spanwise correlation lengths for $\mathrm{L} / \mathrm{D}=1.435$ and $\mathrm{L} / \mathrm{D}=3.70$ are almost $50 \%$ less than that for the single cylinder at this location. Because the correlation length indicates the effective source length along the cylinder axis, the shorter length associated with the tandem configurations could impact radiated noise levels.

Table 1. Vortex shedding frequencies and computed Strouhal numbers.

\begin{tabular}{|c|c|c|}
\hline Configuration & Shedding Frequency $(f), \mathrm{Hz}$ & Strouhal Number $(\mathrm{St})$ \\
\hline Single Cylinder & 365.6 & .290 \\
\hline $\mathrm{L} / \mathrm{D}=1.435$ & 353.1 & .280 \\
\hline $\mathrm{L} / \mathrm{D}=3.70$ & 303.1 & .241 \\
\hline
\end{tabular}




\section{Conclusion}

A joint experimental and computational study has been conducted to characterize the unsteady structures associated with flow interaction between two cylinders in tandem arrangement. Transition strips applied to the upper and lower surface of the front cylinder were successful in achieving turbulent flow separation, thus making the flow field associated with these configurations more representative of the interaction between various components on an aircraft landing gear.

Surface pressures and instantaneous velocities obtained from PIV images were used to examine the mean and instantaneous flow structure for a single cylinder and tandem configurations with $\mathrm{L} / \mathrm{D}=1.435$ and $\mathrm{L} / \mathrm{D}=3.70$. In addition, vortex shedding frequencies and correlation lengths were obtained using a hot wire. Details about the gap flow and configuration wake are summarized below.

1. The gap flow for $\mathrm{L} / \mathrm{D}=1.435$ is asymmetric about the centerline. A recirculation region forms near the lower shear layer and transports vortical structures from the shear layer along the forward surface of the rear cylinder.

2. The gap flow for $\mathrm{L} / \mathrm{D}=3.70$ features shedding from the front cylinder. Vortical structures in the shear layer are convected downstream and interact with the forward surface of the rear cylinder.

3. The configuration wake for $L / D=1.435$ is very similar that of a single cylinder except the wake is narrower and the flow separation occurs further aft on the cylinder. This configuration appears to act as a single bluff body with shedding from the rear cylinder only.

4. The configuration wake for $\mathrm{L} / \mathrm{D}=3.70$ features high TKE levels with $1.5 \mathrm{D}$ of the rear cylinder. The interaction of the fluctuating velocity components with the downstream surface of the rear cylinder could contribute to higher pressure fluctuations.

5. Flow angularity, flow uniformity, blockage, and other tunnel conditions may cause asymmetric flow patterns in the gap and configuration wake at small cylinder spacing.

6. Compared to a single cylinder, spanwise correlation lengths in the wake region of the tandem configurations appear to be significantly shorter.

7. PIV measurements were able to confirm the existence of vortical structures within the fully turbulent shear layers. These structures are convected into the gap and downstream of the rear cylinder where they become part of a shed vortex or diffuse.

Primary input for acoustic models will include lift fluctuations on the cylinder surface. Although PIV measurements do not provide this information, CFD could be used as the bridge between the present measurements and radiated noise predictions. Future work will extend these measurements to tandem cylinders with significantly different diameters.

\section{Acknowledgments}

The authors express their appreciation to Mr. Richard White, Mr. Al Barnes, and Dr. Cheng-Sheng Yao for assisting with the PIV measurements and to Mr. Floyd Backley for the model design and fabrication.

\section{References}

${ }^{1}$ Zorumski, W. E., “Aircraft Noise Prediction Program. Theoretical Manual. Parts 1 and 2,” NASA TM 83199.

${ }^{2}$ Li, F., Khorrami, M. R., and Malik, M. R., "Unsteady Simulation of a Landing-Gear Flow Field,” AIAA-2002-2411, 2002.

${ }^{3}$ Lopes, L. V., Brentner, K. S., Morris, P. J., Lilley, G. M. and Lockard, D. P., "Complex Landing Gear Noise Prediction Using a Simple Toolkit," AIAA-2005-1202, 2005.

${ }^{4}$ Lee, T. and Basu, S., "Nonintrusive Measurements of the Boundary Layer Developing on a Single and Two Circular Cylinders," Experiments in Fluids, 23, 1997, pp. 187-192.

${ }^{5}$ Itoh, Y. and Himeno, R., "Numerical Simulation of Three-Dimensional Flow Around Two Circular Cylinders in Tandem Arrangement," Focused on High Performance Computing in RIKEN 2001, RIKEN Review No. 48, October, 2002.

${ }^{6}$ Alam, M. M., Moriya, M., Takai, K., and Sakamoto, H., "Fluctuation Fluid Forces Acting on Two Circular Cylinders in a Tandem Arrangement at a Subcritical Reynolds Number," Journal of Wind Engineering and Industrial Aerodynamics, 91, 2003, pp. 139-154.

${ }^{7}$ Zdravkovich, M. M., "Review of Flow Interference Between Two Circular Cylinders in Various Arrangements," Journal of Fluids Engineering, Vol. 99, Dec. 1977, pp 618-633. 
${ }^{8}$ Zdravkovich, M.M., "Flow Induced Oscillations of Two Interfering Circular Cylinders," J. of Sound and Vibrations, Vol. 101, No.4, pp. 511-521, 1985.

${ }^{9} \mathrm{Gu}, \mathrm{Z}$. ., "On Interference Between Two Circular Cylinders at Supercritical Reynolds Number," Journal of Wind Engineering and Industrial Aerodynamics, 62, 1996, pp. 175-190.

${ }^{10}$ Lin, J.-C., Yang, Y. And Rockwell, D., Flow Past Two Cylinders in Tandem: Instantaneous and Average Flow Structure," Journal f Fluids and Structures, Vol. 16, Issue 8, 2002, pp. 1059-1071.

${ }^{11}$ Sellers, W. L. and Kjelgaard, S. O., "The Basic Aerodynamic Research Tunnel - A Facility Dedicated to Code Validation," AIAA-88-1997, May 1988.

${ }^{12}$ Washburn, A. E., "The Effect of Freestream Turbulence on the Vortical Flow over a Delta Wing," Master of Science Thesis, George Washington University, 1990.

${ }^{13}$ Jenkins, L. N., "An Experimental Investigation of the Flow Over a Notchback Automobile Configuration," Master of Science Thesis, George Washington University, 1999.

${ }^{14}$ Hama, F.R., “An Efficient Tripping Device," Journal of Aeronautical Sciences, March, 1957.

${ }^{15}$ Hegarty, J. C. and Hama, F. R., "Further Investigations on the Triangular-Patch Stimulator," AFOSR TN-57-616, June, 1957.

${ }^{16}$ Roshko, A. "Experiments on the Flow Past a Circular Cylinder At Very High Reynolds Number," Journal of Fluid Mechanics, Vol. 10, 1961, pp 345-356.

${ }^{17}$ Lourenco, L. M. and Krothapalli, A., "True Resolution PIV: A Mesh-Free Second Order Accurate Algorithm," Proceedings of the 10th International Symposium on Application Techniques in Fluid Mechanics, Lisbon, July 2000.

${ }^{18}$ Khorrami, M. R., Choudhari, M. M., Jenkins, L. N. and McGinley, C. B., "Unsteady Flowfield Around Tandem Cylinders as Prototype for Component Interaction in Airframe Noise," AIAA-2005-2866, 2005.

${ }^{19}$ Couregelongue, J., "On the existence of two families of eddies behind immersed solids," Comptes Rendus Academie Sciences, Vol. 189, pp. 972-974, 1929. 


\section{Figures}

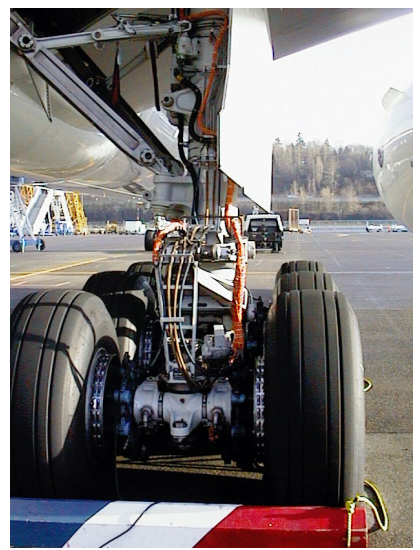

Figure 1. Image showing landing gear complexity.
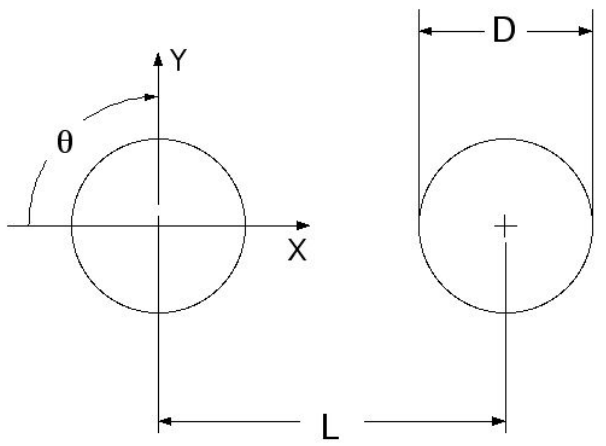

Figure 3. Model schematic and coordinate reference system.

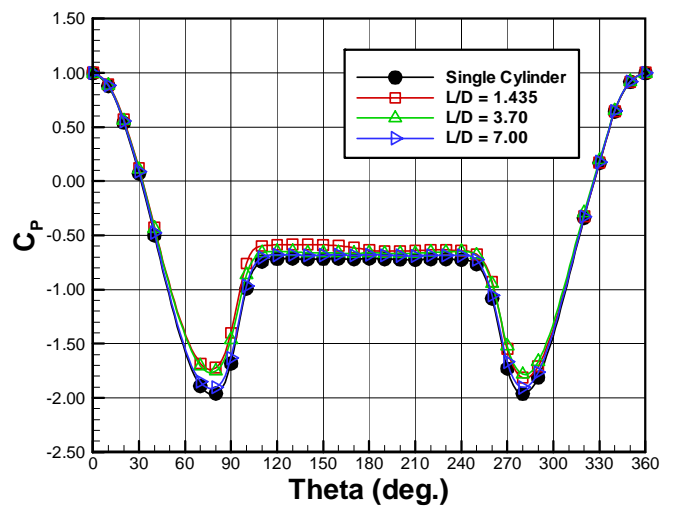

Figure 5. Pressure distribution on front cylinder.

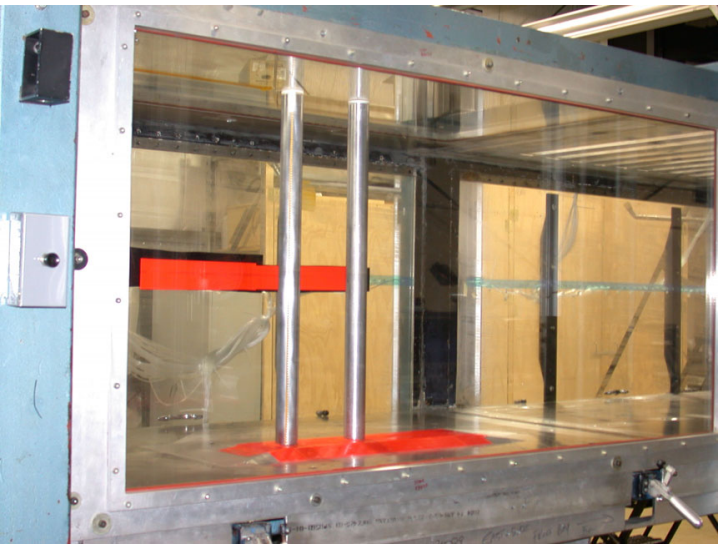

Figure 2. Tandem cylinder configuration in the BART facility.

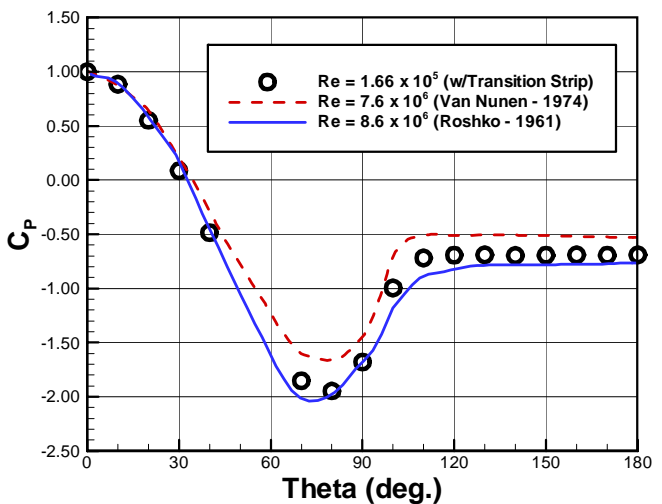

Figure 4. Transition strip effectiveness

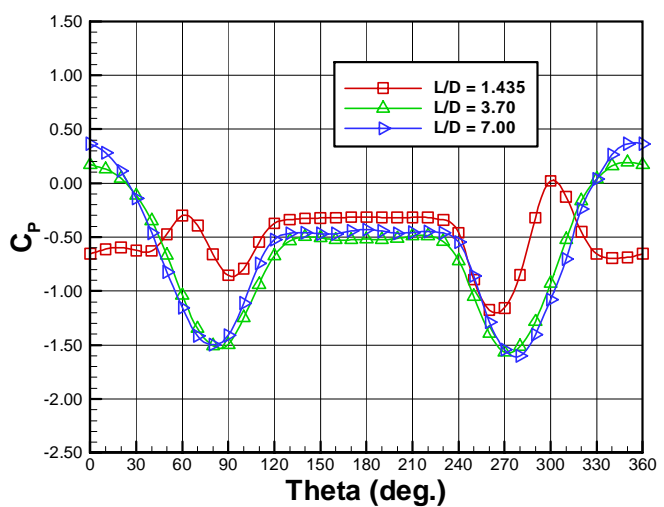

Figure 6. Pressure distribution on downstream cylinder. 


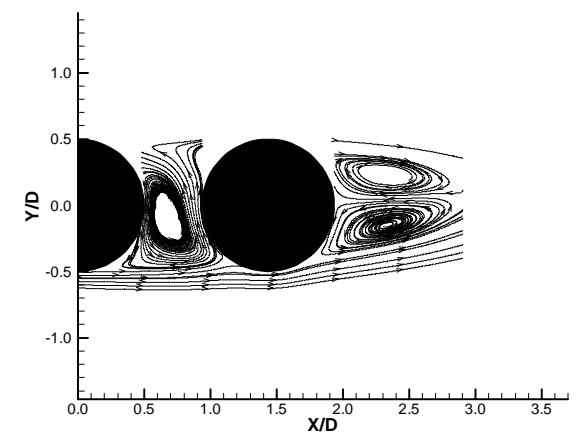

Figure 7a. Mean streamlines $(\mathrm{L} / \mathrm{D}=1.435)$

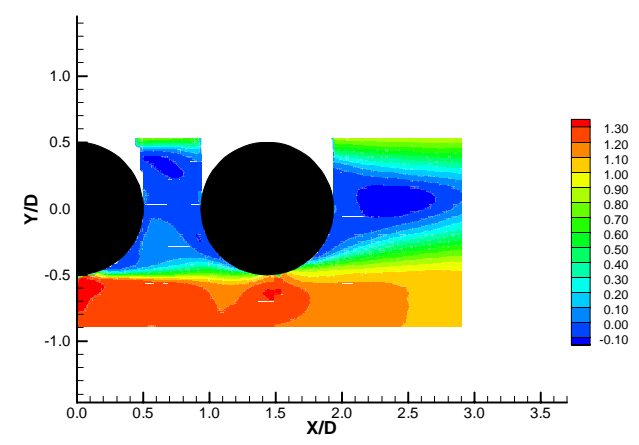

Figure 7b. Mean streamwise velocity $(L / D=1.435)$

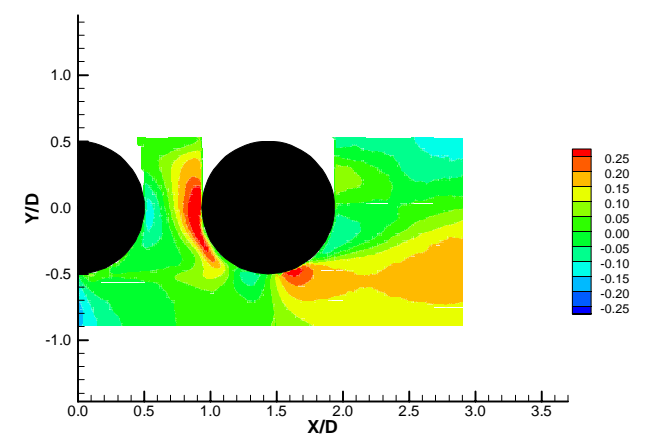

Figure 7c. Mean vertical velocity $(L / D=1.435)$

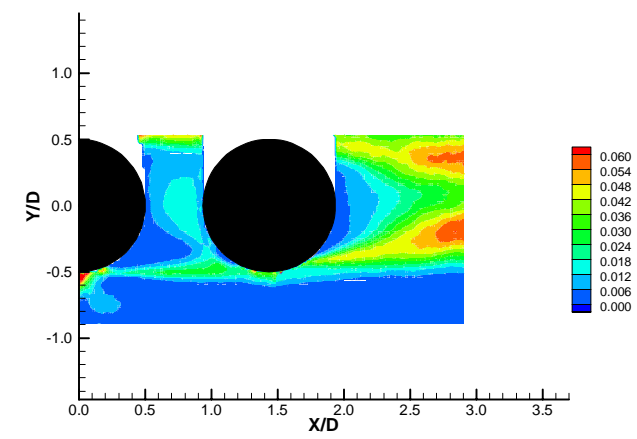

Figure 7d. Turbulent kinetic energy $(L / D=1.435)$

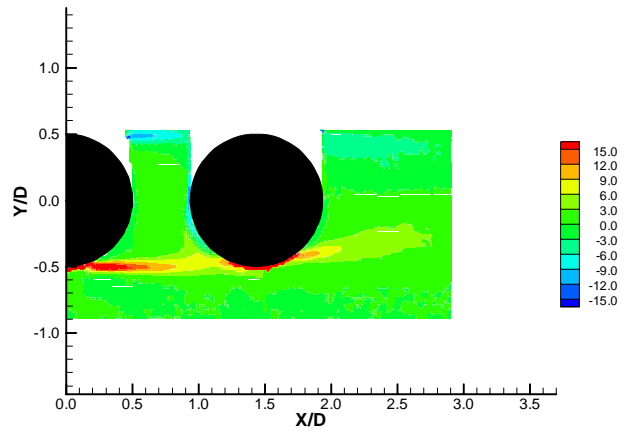

Figure 7e. Mean spanwise vorticity $(L / D=1.435)$

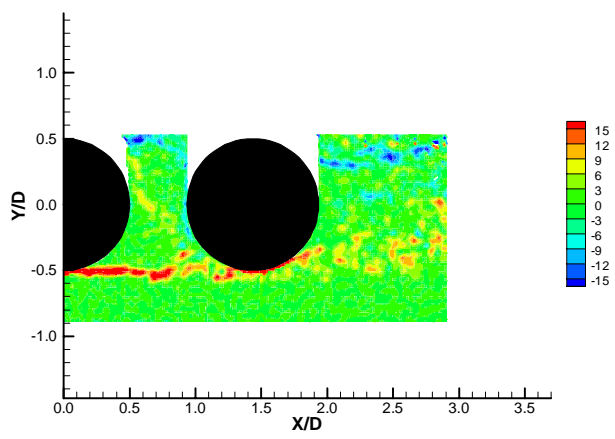

Figure 7f. Instantaneous spanwise vorticity (Frame 4, $L / D=1.435)$

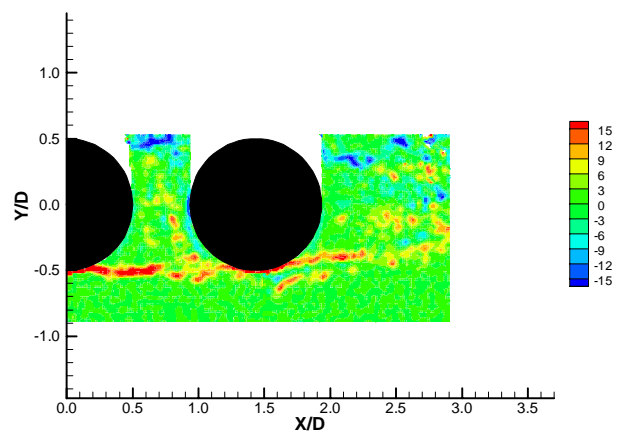

Figure 7g. Instantaneous spanwise vorticity (Frame $10, \mathrm{~L} / \mathrm{D}=1.435$ )

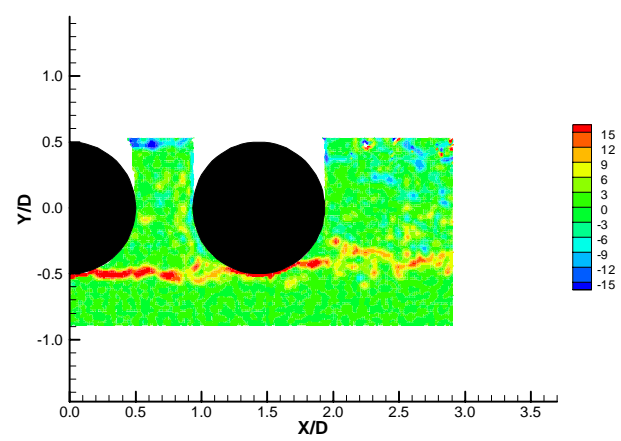

Figure $7 \mathrm{~h}$. Instantaneous spanwise vorticity (Frame $8, L / D=1.435$ )

10

American Institute of Aeronautics and Astronautics 


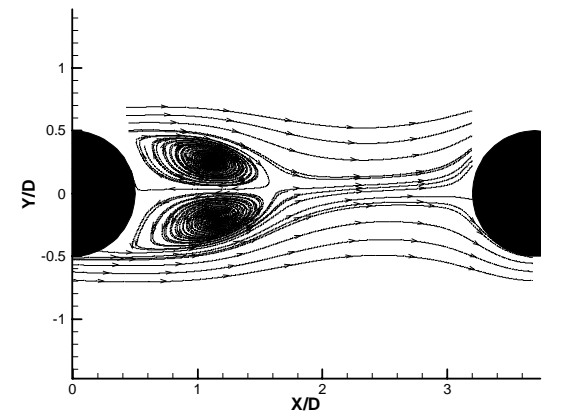

Figure 8a. Mean streamlines $(1 / \mathrm{D}=3.70)$

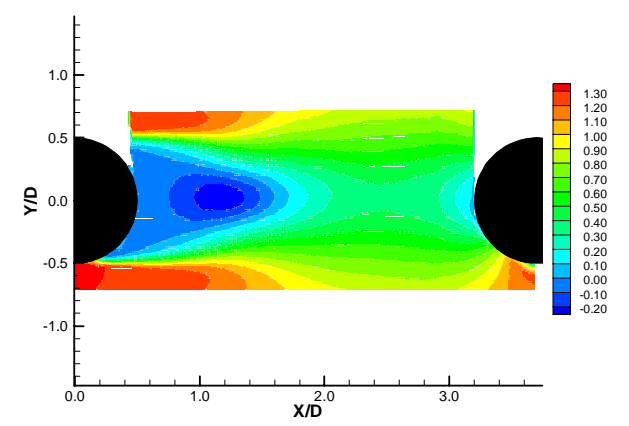

Figure 8b. Mean streamwise velocity $(L / D=3.70)$

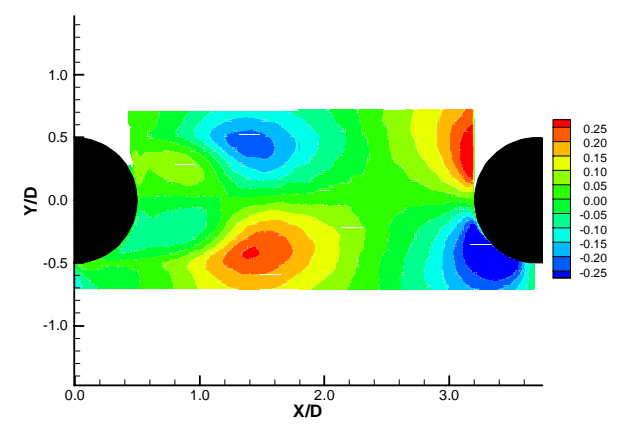

Figure 8c. Mean vertical velocity $(L / D=3.70)$

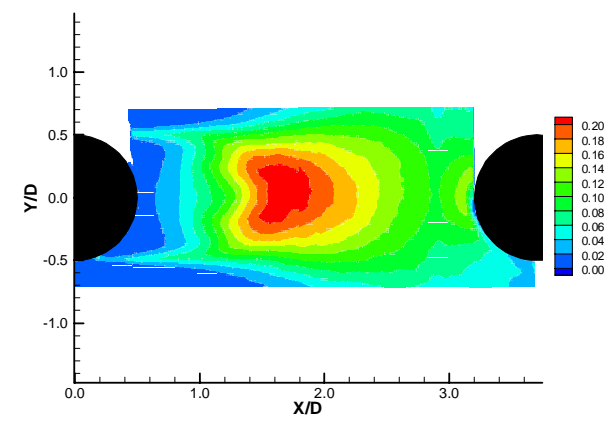

Figure 8d Turbulent kinetic energy $(L / D=3.70)$

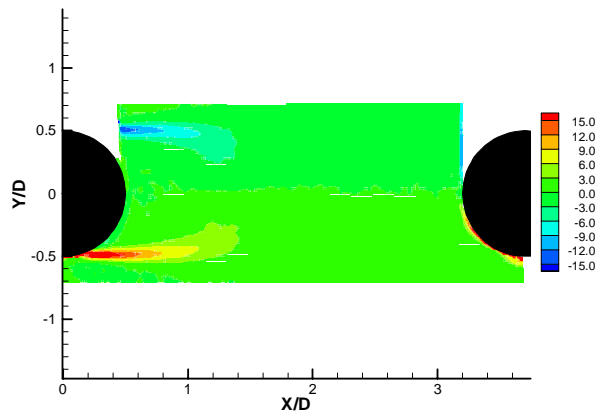

Figure 8e Mean spanwise vorticity $(L / D=3.70)$

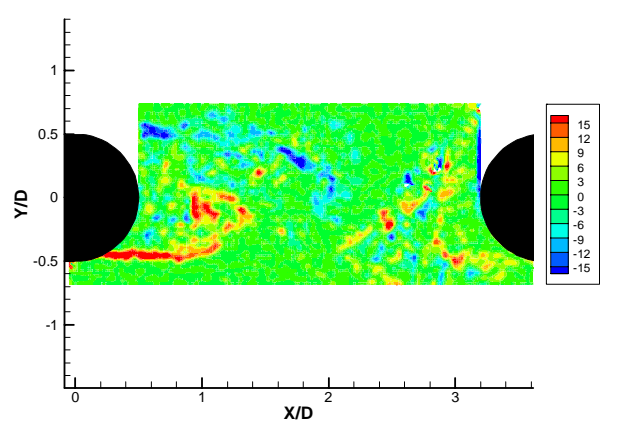

Figure 8f. Instantaneous spanwise vorticity (Frame 16, $\mathrm{L} / \mathrm{D}=3.70$ )

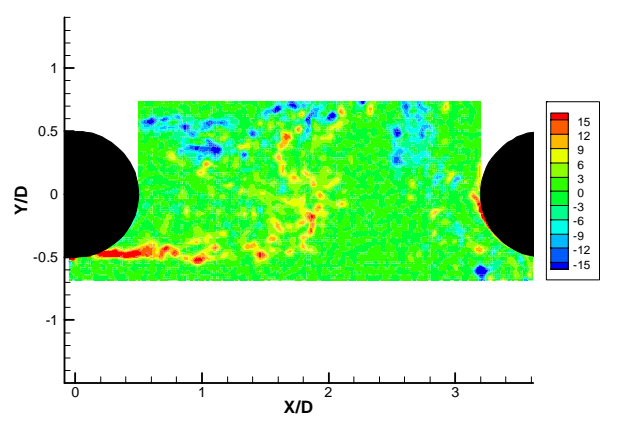

Figure 8g. Instantaneous spanwise vorticity (Frame $4, \mathrm{~L} / \mathrm{D}=3.7)$

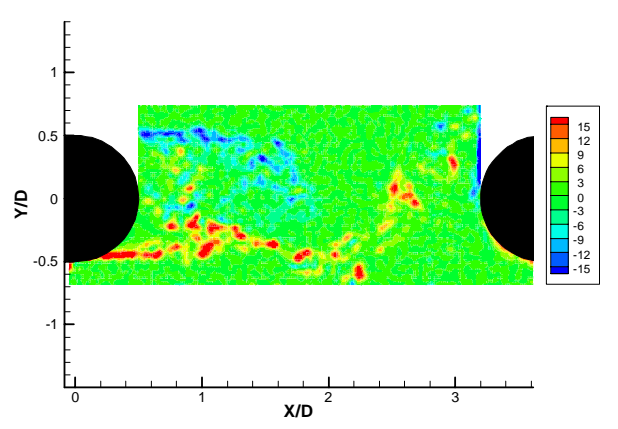

Figure 8h. Instantaneous spanwsie vorticity (Frame $19, \mathrm{~L} / \mathrm{D}=3.70$ ) 


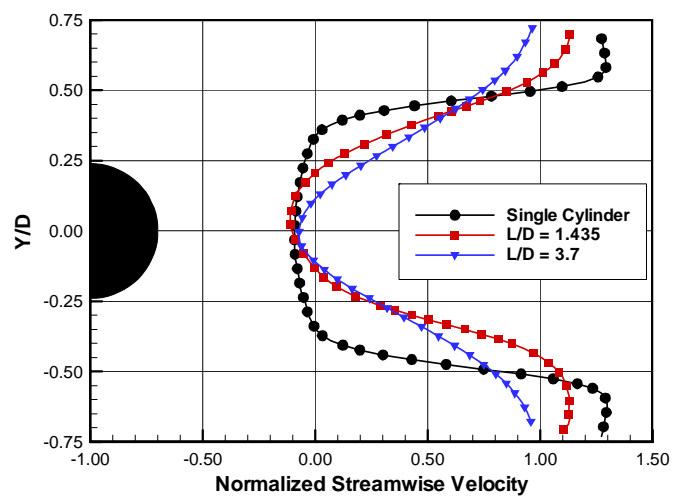

Figure 9a. Mean streamwise velocity profiles at $\mathrm{X} / \mathrm{D}$ $=0.75$.

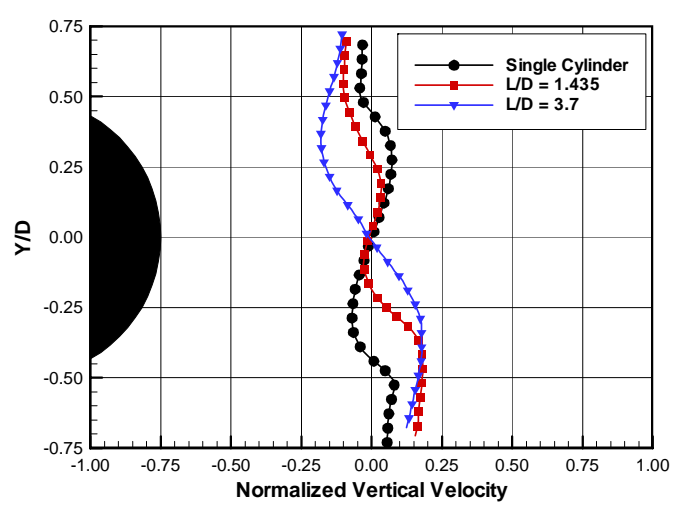

Figure 9b. Mean vertical velocity profiles at $\mathrm{X} / \mathrm{D}=\mathbf{0 . 7 5}$.

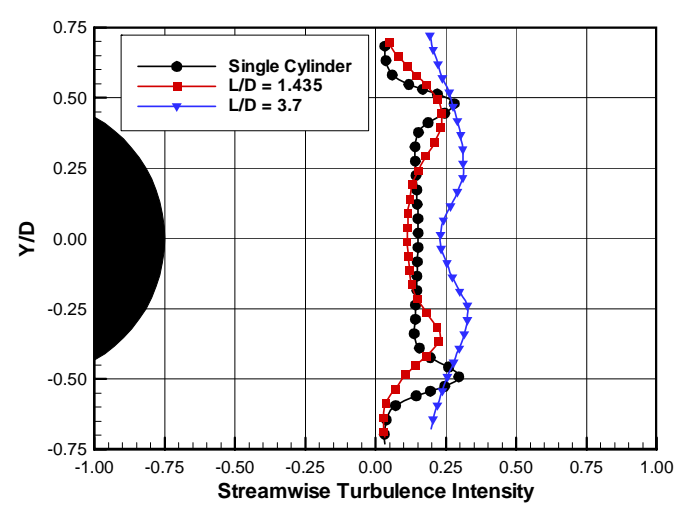

Figure 9c. Streamwise turbulence intensity at $\mathrm{X} / \mathrm{D}=\mathbf{0 . 7 5}$.

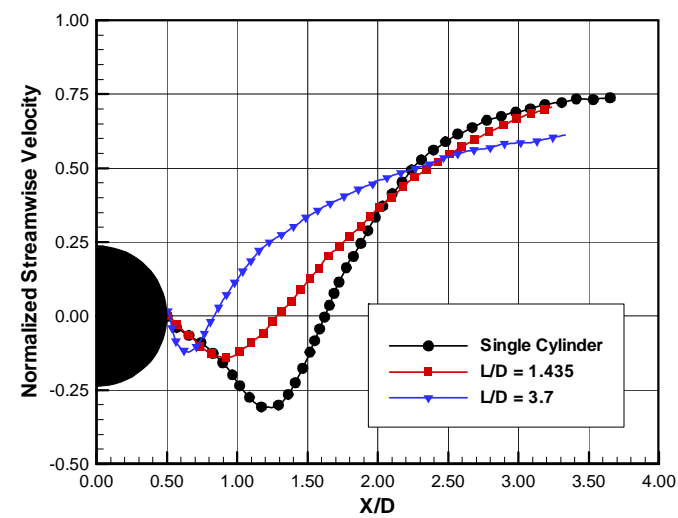

Figure 10a. Mean streamwise velocity along centerline downstream of single/rear cylinder

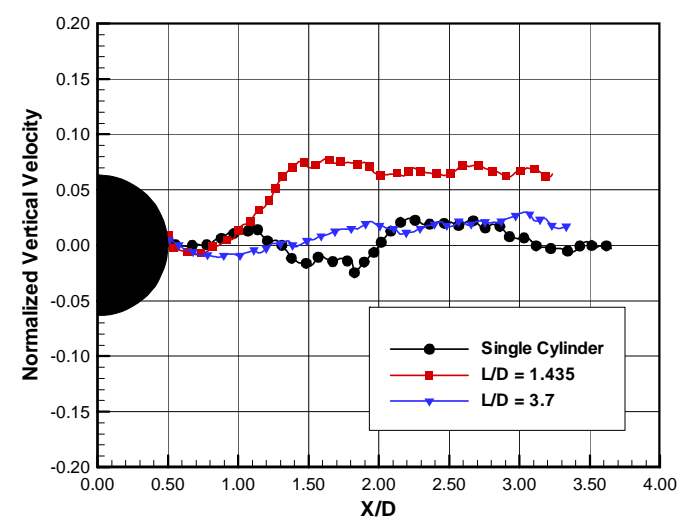

Figure 10b. Mean vertical velocity along centerline downstream of single/rear cylinder

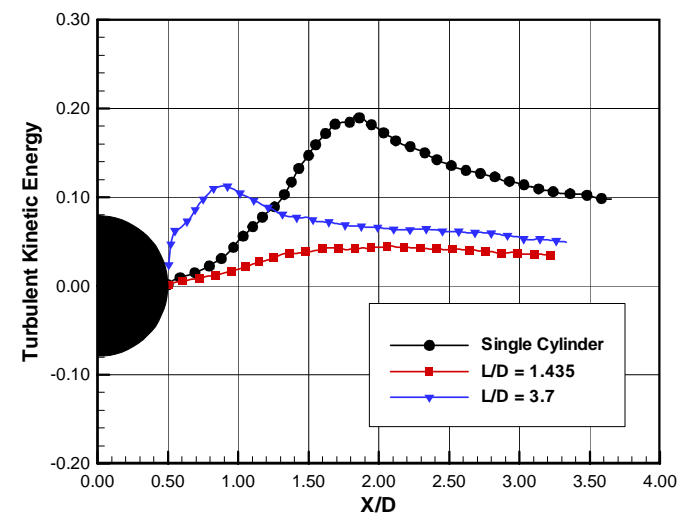

Figure 10c. Turbulent kinetic energy (TKE) along centerline downstream of single/rear cylinder 


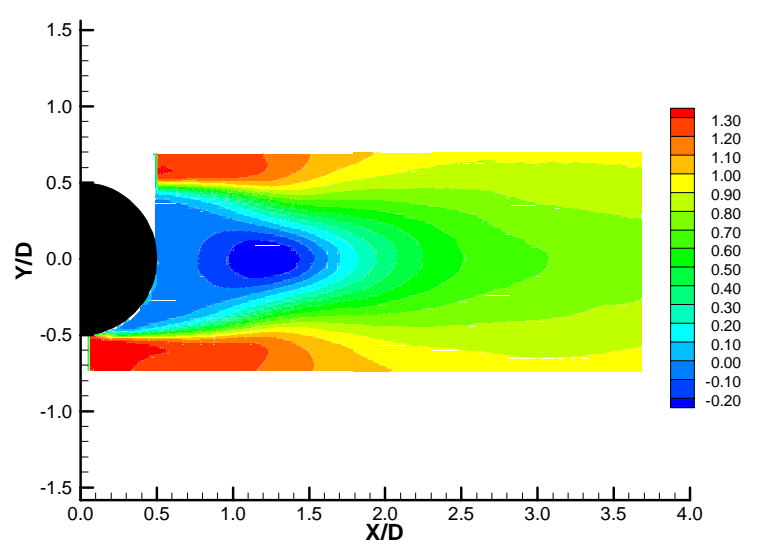

Figure 11a. Mean streamise velocity (single cylinder)

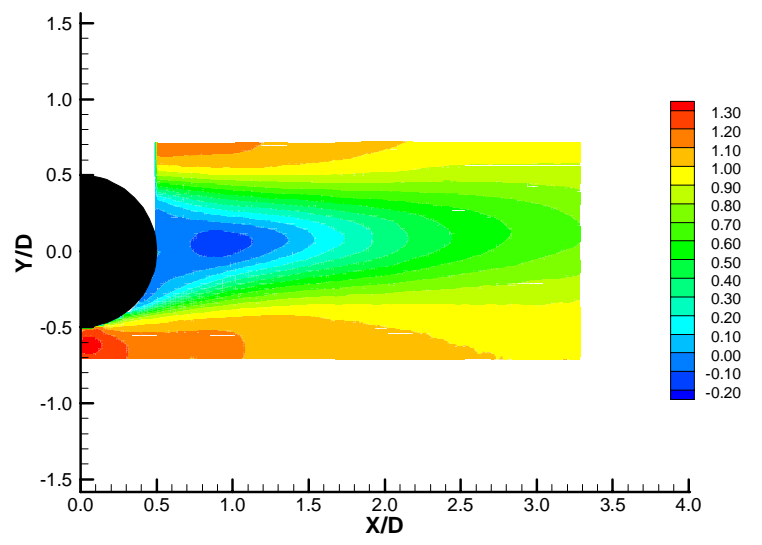

Figure 11b. Mean streamise velocity $(L / D=1.435)$

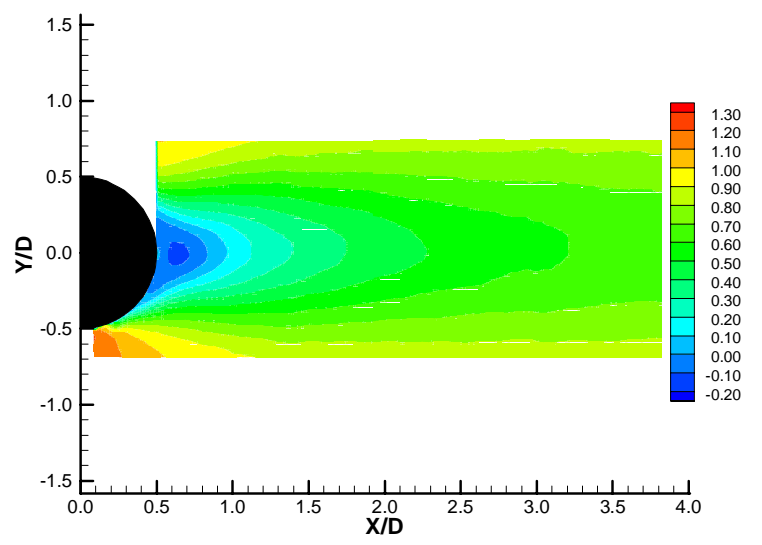

Figure 11c. Mean streamise velocity $(L / D=3.70)$

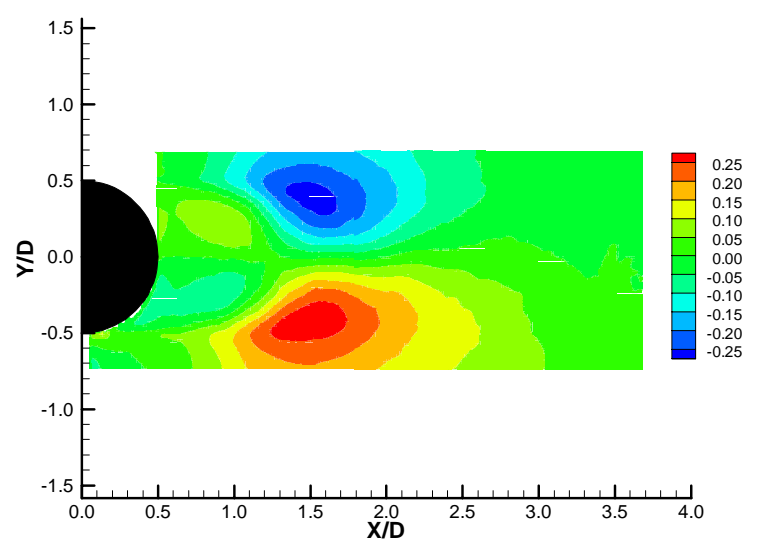

Figure 12a. Mean vertical velocity (single cylinder)

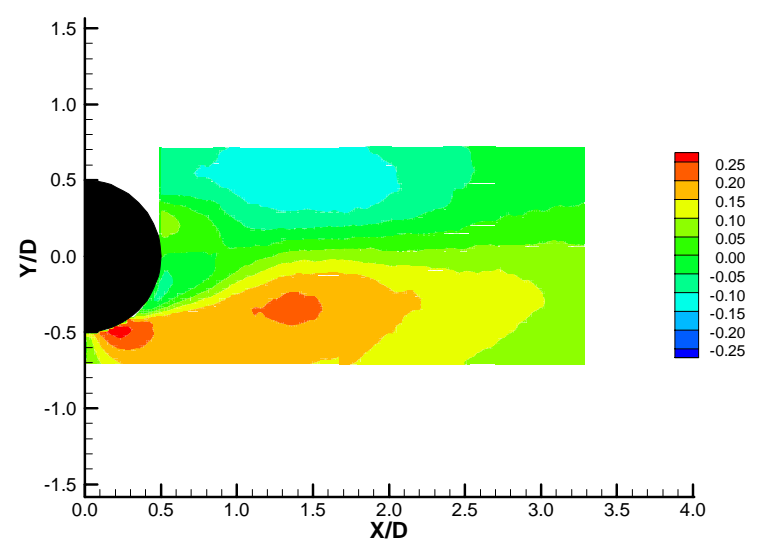

Figure 12b. Mean vertical velocity $(L / D=1.435)$

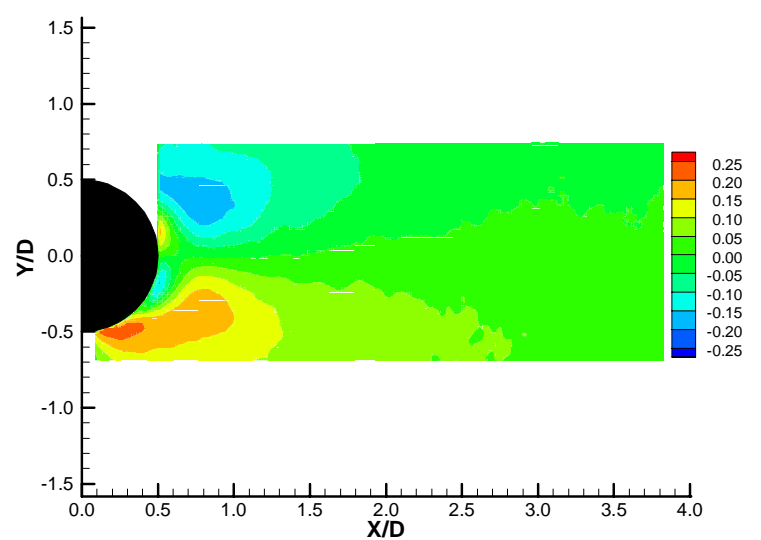

Figure 12c. Mean vertical velocity $(L / D=3.70)$ 


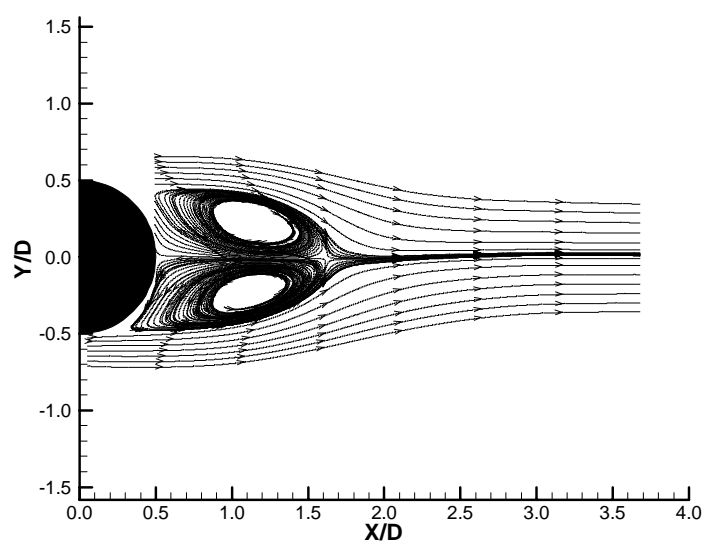

Figure 13a. Mean flow streamlines (single cylinder)

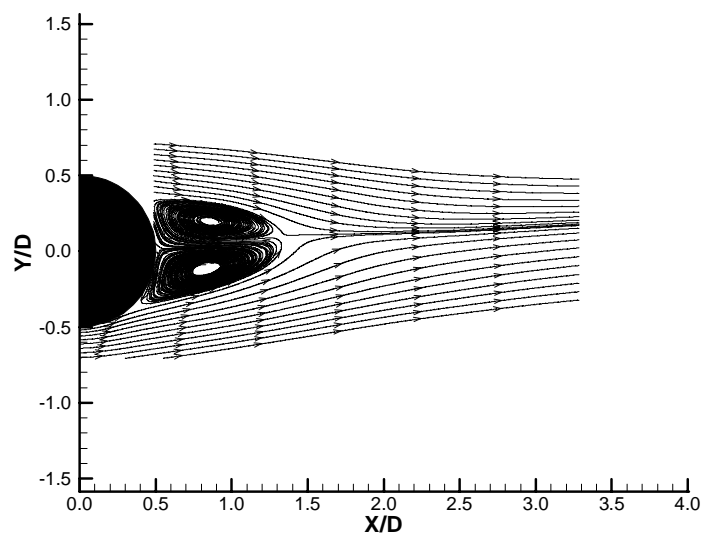

Figure 13b. Mean flow streamlines $(\mathrm{L} / \mathrm{D}=1.435)$

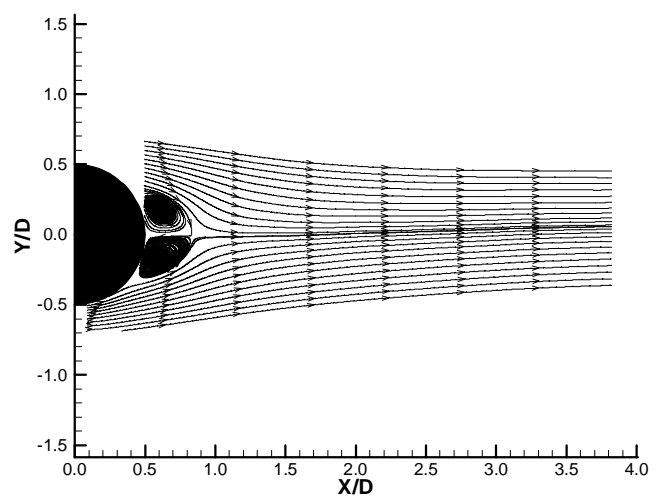

Figure 13c. Mean flow streamlines $(\mathrm{L} / \mathrm{D}=3.70)$

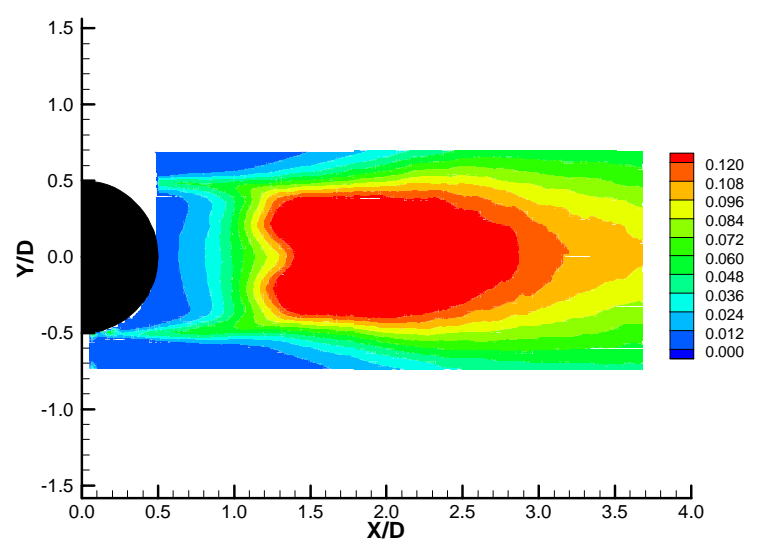

Figure 14a. Turbulent kinetic energy (single cylinder)

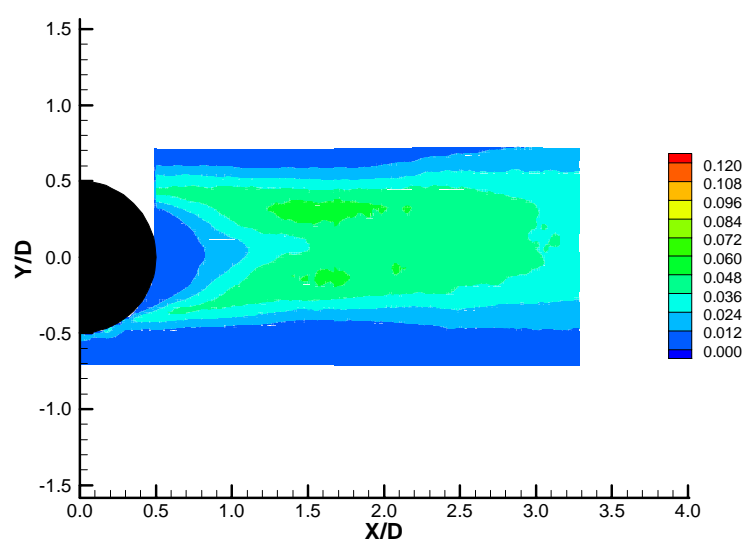

Figure 14b. Turbulent kinetic energy $(\mathrm{L} / \mathrm{D}=1.435)$

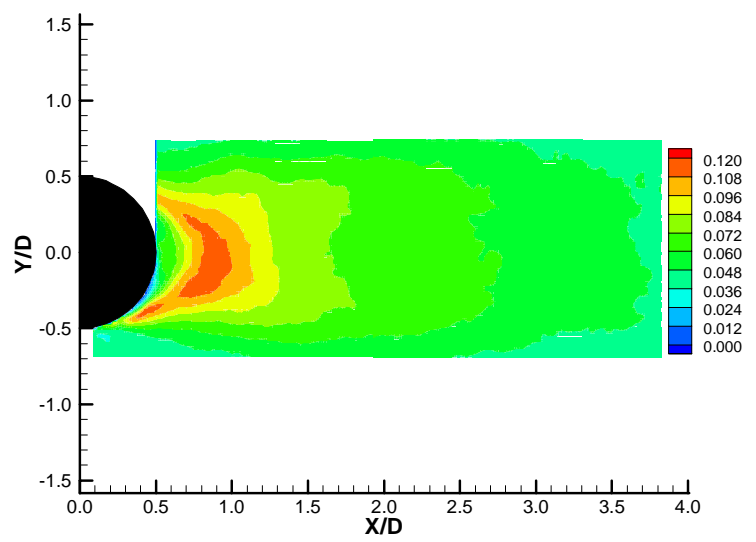

Figure 14c. Turbukent kinetic energy $(\mathrm{L} / \mathrm{D}=3.70)$ 


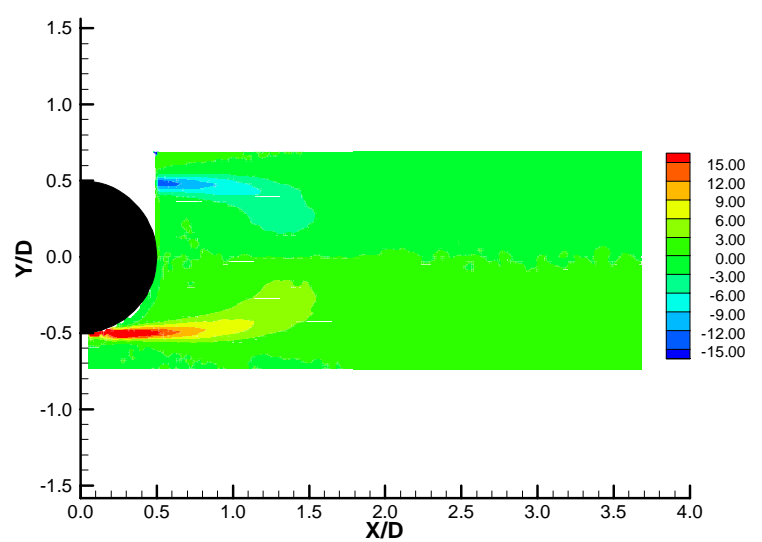

Figure 15a. Mean spanwise vorticity (single cylinder)

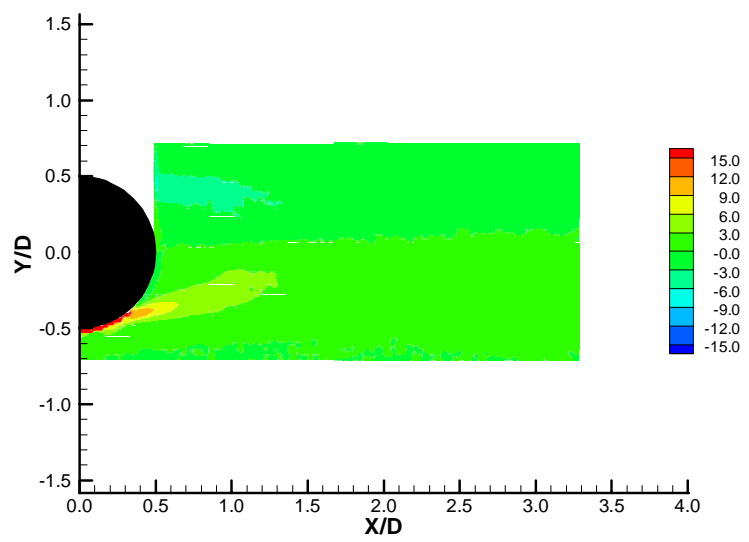

Figure 15b. Mean spanwise vorticity $(L / D=1.435)$

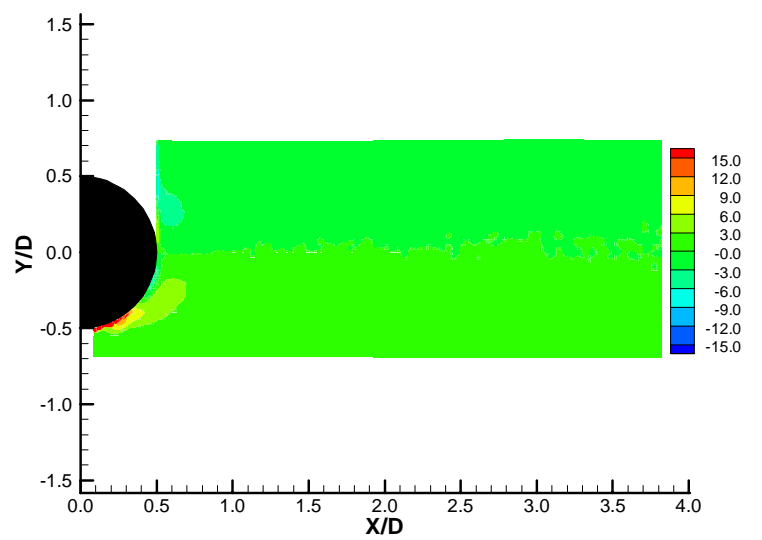

Figure 15c. Mean spanwise vorticity $(L / D=3.70)$

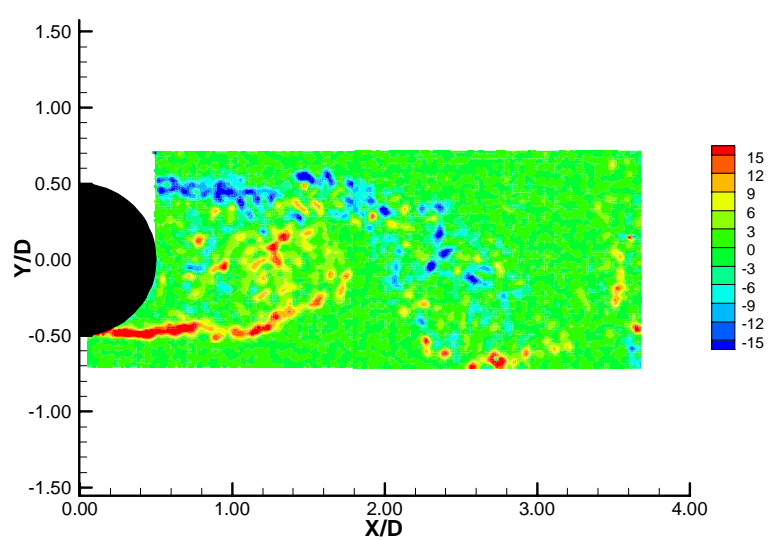

Figure 16a. Instantaneous spanwise vorticity (Single cylinder)

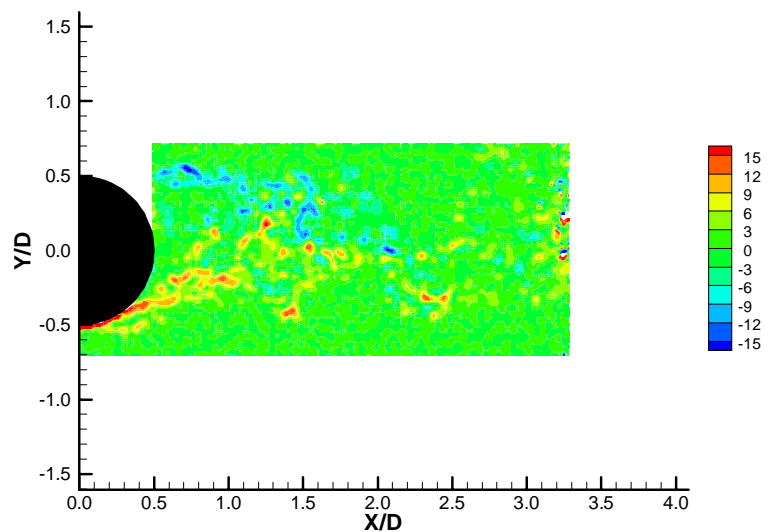

Figure 16b. Instantaneous spanwise vorticity $(\mathrm{L} / \mathrm{D}=$ 1.435)

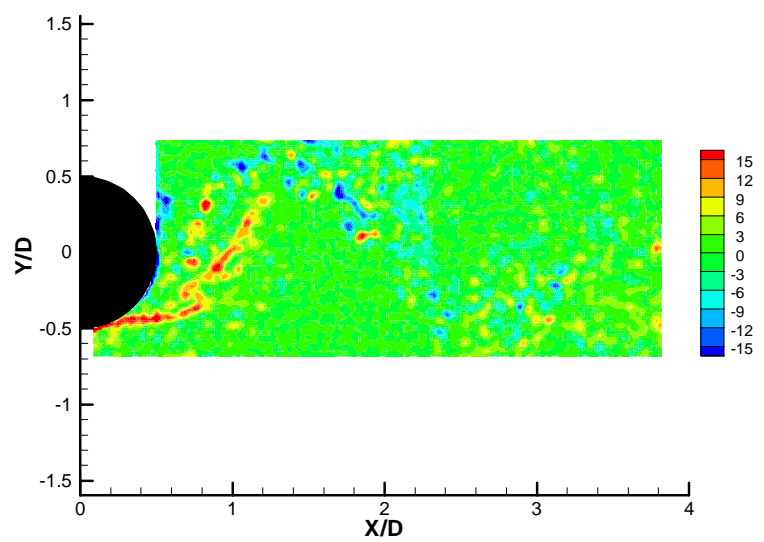

Figure 16c. Instantaneous spanwise vorticity $(\mathrm{L} / \mathrm{D}=$ 3.70) 


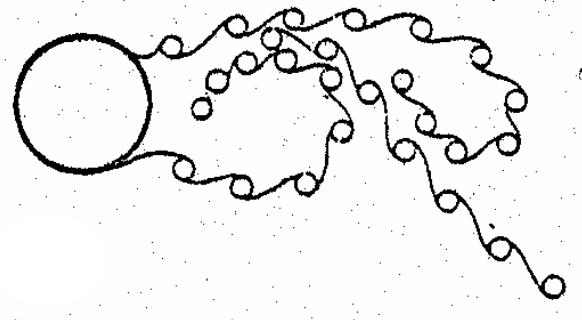

Figure 17. Transition eddies in shear layer Figure 19. Velocity spectra. (Couregelongue 1929)

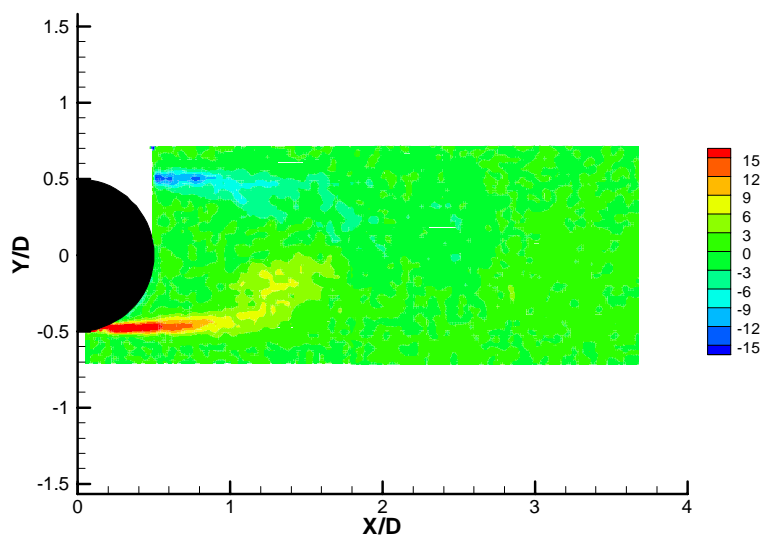

Figure 18. Phase-averaged vorticity (single cylinder)

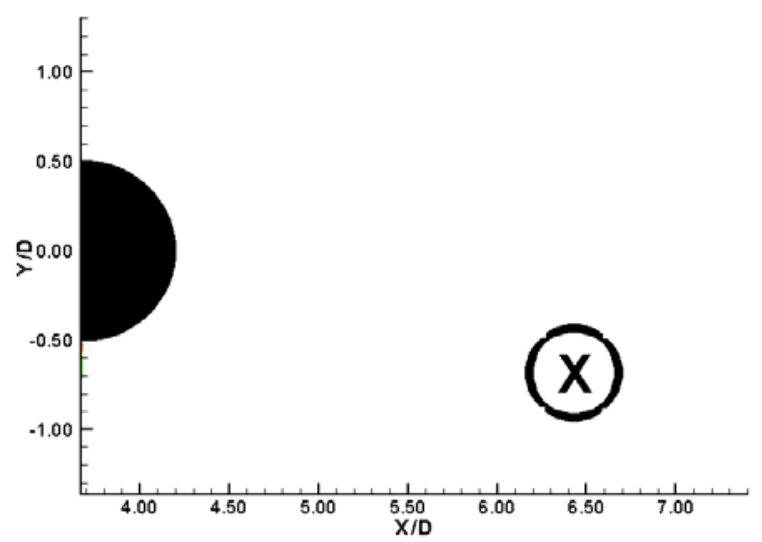

Figure 19. Velocity spectra measurement location.
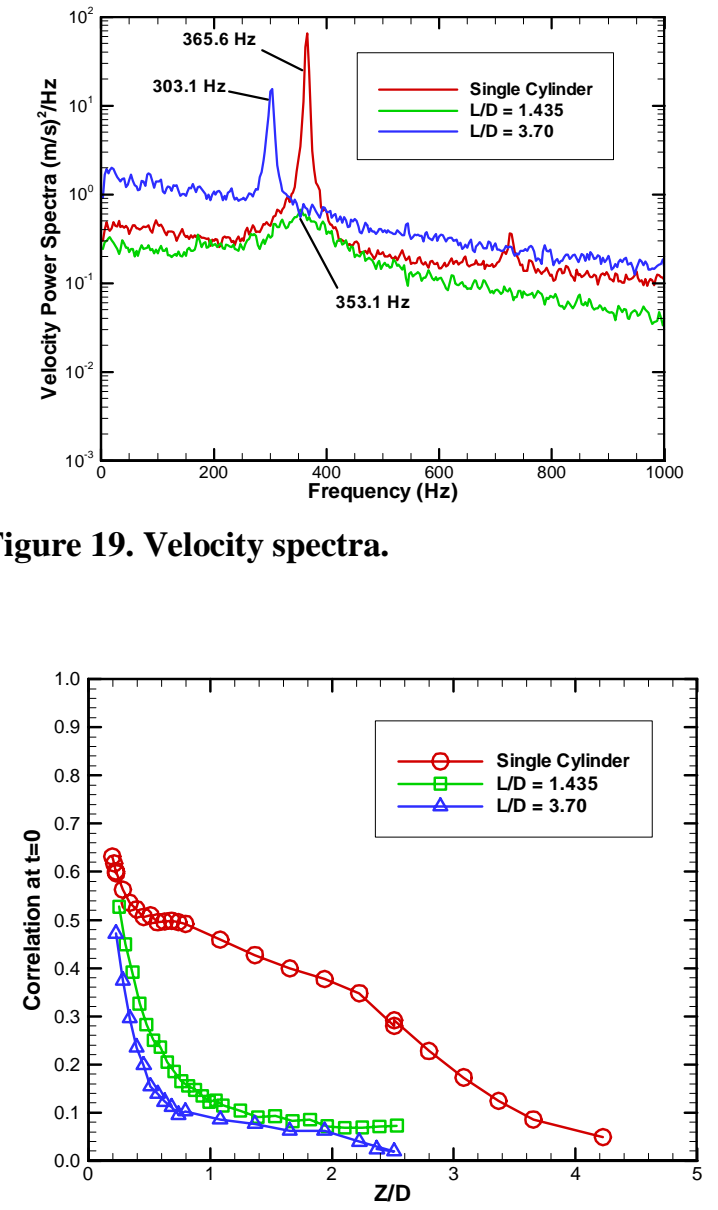

Figure 20. Spanwise correlation. 